\title{
6.7 GHz methanol masers at sites of star formation
}

\section{A blind survey of the Galactic plane between $20^{\circ} \leq l \leq 40^{\circ}$ and $|b| \leq 0.52$}

\author{
M. Szymczak, A. J. Kus, G. Hrynek, A. Kȩpa, and E. Pazderski
}

Toruń Centre for Astronomy, Nicolaus Copernicus University, Gagarina 11, 87-100 Toruń, Poland

Received 30 April 2002 / Accepted 6 June 2002

\begin{abstract}
We report the results of an unbiased survey for $6.7 \mathrm{GHz}$ methanol maser emission of a $\sim 21 \mathrm{deg}^{2}$ strip of the Galactic plane carried out with the $32 \mathrm{~m}$ Torun radio telescope. An area at $20^{\circ} \leq l \leq 40^{\circ},|b| \leq 0.52$ was surveyed in an equilateral triangular grid with a sensitivity limit of about $1.6 \mathrm{Jy}$. We detected a total of 100 sources, 26 of which are new detections. All the new sources are of moderate intensity and their peak flux densities have median value of $6.5 \mathrm{Jy}$, i.e. about half that of previously known sources in the sample. About $80 \%$ of maser sources have IR counterpart candidates within a $1^{\prime}$ radius but not all the IRAS counterparts of methanol masers have colours typical of ultracompact HII regions. An excess of masers unassociated with IR sources occurs at $30^{\circ}<l<35^{\circ}$ because of incompleteness of IR catalogues due to strong confusion near the tangential region of the spiral arm. Our unbiased survey doubled the number of detections as compared to IRAS-based observations. Within the positional uncertainty of $1^{\prime}$ about one third of the methanol sources have radio continuum counterparts at $5 \mathrm{GHz}$ of a flux density greater than $2.5-10 \mathrm{mJy}$. The distribution of methanol sources appears to be consistent with a clustered mode of formation of massive stars.
\end{abstract}

Key words. masers - surveys - stars: formation - ISM: molecules - radio lines: ISM - HII regions

\section{Introduction}

Central to the use of masers in studies of molecular clouds with star-forming activity have been the surveys that have provided large samples for subsequent investigations. The $6.7 \mathrm{GHz}$ methanol transition (Menten 1991) has recently joined the $\mathrm{OH}$ and $\mathrm{H}_{2} \mathrm{O}$ masers as a powerful tool in identifying the sites of massive stars, which are probably at the earliest stages of evolution, and in studying the structure of their environments. A large survey in the $\mathrm{CH}_{3} \mathrm{OH}$ maser line performed by Caswell et al. (1995) mostly towards southern OH objects or other sites of star formation has resulted in the detection of 245 sources.

Several new detections were made in extensive surveys of IRAS selected samples of ultracompact HII (UCHII) region candidates (Schutte et al. 1993; van der Walt et al. 1995; 1996; Walsh et al. 1997; MacLeod et al. 1998; Slysh et al. 1999; Szymczak et al. 2000). In the sample of 535 southern UCHII candidates searched by Walsh et al. (1997) maser emission at $6.7 \mathrm{GHz}$ occurred in 201 objects. For the sample of 1399 northern UCHII candidates with much less stringent criteria (Wood \& Churchwell 1989; Hughes \& MacLeod 1989) imposed for IRAS colours, 182 methanol masers were found (Szymczak et al. 2000). These studies clearly demonstrate that

Send offprint requests to: M. Szymczak, e-mail:msz@astro.uni.torun.pl the detection rate strongly depends on the IRAS colours; in fact almost all UCHII region candidates with extremely red colours are $6.7 \mathrm{GHz}$ maser sources. This can imply a close relationship between the occurrence of $\mathrm{CH}_{3} \mathrm{OH}$ maser phenomena and the evolutionary stage of powering stars. The effect of the farinfrared flux density and the galactic location on the methanol detection rate is also well documented (e.g. Szymczak \& Kus 2000). However, they have shown that about $18 \%$ of methanol sources have IRAS colours well outside the limits established for UCHII regions by Wood \& Churchwell (1989). This result may suggest a population of $6.7 \mathrm{GHz}$ masers not associated with typical regions of massive star formation. A support for this view has been provided by the unbiased survey of the region of the Galactic plane by Ellingsen et al. (1996b). They found that many $6.7 \mathrm{GHz}$ methanol masers are not associated with IRAS sources, while some maser sources have colours atypical for the UCHII regions. The observations with high angular resolution also revealed that several $6.7 \mathrm{GHz}$ maser sites are not associated with or are offset from the UCHII regions (Ellingsen et al. 1996a; Phillips et al. 1998; Walsh et al. 1998). It has been postulated that some methanol sources associated with OB star formation appear before the UCHII regions form or they are associated with less massive protostars (Caswell 1996; Ellingsen et al. 1996b). 
Here we present the results of the first part of a blind survey of the Galactic plane, unbiased by previous infrared information. Such a survey would be productive to enhance the sample of $6.7 \mathrm{GHz}$ masers and helpful to elucidate the general problems outlined above. It can be a starting point for comprehensive studies of individual objects by other means.

\section{Observations}

The observations were carried out using the Torun $32 \mathrm{~m}$ telescope over a number of sessions between February and October 2000. The half-power beam width (HPBW) of the antenna at $6.7 \mathrm{GHz}$ was 5.5. The telescope was equipped with a dual-channel HEMT receiver accepting two circular polarizations. The system noise level was about 350 Jy under good weather conditions. The backend was a $2^{14}$-channel autocorrelation spectrometer operating in 2-bit mode. We used it in two parts of 4096 channels each with a bandwidth of $4 \mathrm{MHz}$. The resulting spectral resolution was $0.04 \mathrm{~km} \mathrm{~s}^{-1}$ and the velocity coverage was $\pm 90 \mathrm{~km} \mathrm{~s}^{-1}$. The bandwidth was always centred at the local standard of rest velocity of $40 \mathrm{~km} \mathrm{~s}^{-1}$.

The total area of $20.8 \mathrm{deg}^{2}$, in the longitude range from $20^{\circ}$ to $40^{\circ}$ and latitude range from -0.52 to 0.52 , was surveyed in an equilateral triangular grid pattern. The separation of each point of this grid from all adjacent points was 4.4 (0.8 the HPBW at $6.668518 \mathrm{GHz}$ ) and about 4800 sky positions were sampled. The spectra were taken in total power mode with 10 min integration time at each grid point. The spectral line flux densities were continuously calibrated by measuring the receiver response to the signal from a noise diode of known temperature. The behaviour of the system was checked through continuum observations of 3C123 and Vir A assuming flux densities from Ott et al. (1994). The accuracy of the absolute flux density calibration was usually better than $15 \%$.

The approximate position of each maser was estimated in the initial survey. A better position was determined by observing a grid of nine points centred on the approximate position. The accuracy of position measurements of methanol sources was better than $30^{\prime \prime}$ for all objects with a $10 \sigma$ detection or better. For stronger sources the positional accuracy did not improve significantly, as expected from the theoretical value of the order of the HPBW divided by the signal to noise ratio, because it was limited by pointing errors of the antenna of about $25^{\prime \prime}$.

The data were reduced applying standard procedures. The average $3 \sigma$ sensitivity level in the spectra after averaging the two polarizations was $1.6 \mathrm{Jy}$. However, due to a wide variety of observing conditions, $1 \sigma$ varied between $0.4 \mathrm{Jy}$ to $0.7 \mathrm{Jy}$. All sky positions with poor quality spectra $(1 \sigma>0.7 \mathrm{Jy})$ were reobserved in better weather conditions to achieve a sensitivity in the range mentioned above. The absolute radial velocity may have an error of $\pm 0.4 \mathrm{~km} \mathrm{~s}^{-1}$.

To estimate the completeness limit (the peak flux density for which the probability of maser detection is 0.99 ) of the survey we applied a simple approach outlined by Johannson et al. (1977). Using the $3 \sigma$ detection level of $1.6 \mathrm{Jy}$ we calculated that all the sources of peak flux density greater than 5.2 Jy were detected within the surveyed area.

\section{Results}

Table 1 lists the maser sources we did detect; their galactic coordinates used as the name, the equatorial coordinates for epoch J2000 as measured in this work, the velocity range of maser emission $\Delta V$, the velocity of peak emission $V_{\mathrm{p}}$, the peak flux density $S_{\mathrm{p}}$, the integrated flux density $S_{\mathrm{i}}$, the reference of the discovery and the two last columns give the position offset and the name of the associated IR sources discussed in the next section. In total there are 100 sources, 26 of which were detected for the first time. The spectra of all the new sources are presented in Fig. 1.

The new discoveries were relatively weak sources with $S_{\mathrm{p}}$ lower than $28 \mathrm{Jy}$ and the median value of $S_{\mathrm{p}}$ was $6.5 \mathrm{Jy}$. The median peak flux density of the known sources was $12.2 \mathrm{Jy}$ while $S_{\mathrm{p}}$ were in the range of 1.9 to $473.7 \mathrm{Jy}$. The velocity spread for the new sources of median value of $7 \mathrm{~km} \mathrm{~s}^{-1}$ was comparable with $7.5 \mathrm{~km} \mathrm{~s}^{-1}$ observed for the known sources.

\section{Methanol emission and other signposts of star formation}

\subsection{IR sources}

The positional accuracy of maser sources depends upon the peak flux density of the strongest feature used for measurements. For the $32 \mathrm{~m}$ antenna it was usually better than $30^{\prime \prime}$ for sources with the flux density greater than $10 \mathrm{Jy}$. However, it was as poor as $70^{\prime \prime}$ for sources with the flux density of a few Jy. Considering that the pointing errors were about $25^{\prime \prime}$ and the positional uncertainties of IRAS measurements were of about $30^{\prime \prime}$ we cautiously assumed that each maser source could be associated with an IR object that is located within a radius of less than $1^{\prime}$.

We searched the SIMBAD data base for infrared counterparts of our detections. There are 50 maser sources for which IRAS (1986) counterpart candidates have been found within a $1^{\prime}$ radius. A further 29 sources have been identified mainly in the MSX5C catalogue (Egan et al. 1999) using the same matching criterion. The positional differences $\Delta$ pos, are listed in Table 1 together with the name of IR counterpart candidate. Among the rest 21 detections, 15 and 6 objects have IR counterpart candidates, in the IRAS and/or MSX5C catalogues, within radii of 90 and $120^{\prime \prime}$ respectively. Those associations require to be verified by more precise measurements of methanol positions.

In the group of 50 sources associated with IRAS objects within the average positional uncertainty of $28^{\prime \prime}$ there are 20 objects of high quality IRAS measurements at 12,25 and $60 \mu \mathrm{m}$ bands and the colours can be well determined. Figure 2 shows the two colour diagram for all 50 IRAS sources of our methanol detections. Almost all the sources with well determined colours lie inside the colour limits established for UCHII regions (Wood \& Churchwell 1989, hereafter WC89). The colours of only three sources do not satisfy WC89 criteria but two of those, IRAS 18278-1009 and IRAS 18441-0134 satisfy less stringent criteria for UCHII regions (Hughes \& MacLeod 1989, hereafter HM89). There is 
Table 1. $6.7 \mathrm{GHz}$ methanol masers in the region $l=20^{\circ}-40^{\circ},|b|=0.52 . \Delta V$ is the velocity range of maser emission and $V_{\mathrm{p}}$ is the velocity of peak emission. $S_{\mathrm{p}}$ and $S_{\mathrm{i}}$ are the peak flux density and the integrated flux density, respectively. $\Delta$ pos is the position difference between methanol source and its infrared counterpart candidate.

\begin{tabular}{|c|c|c|c|c|c|c|c|c|c|}
\hline Source $(l, b)$ & $\alpha(2000)$ & $\delta(2000)$ & $\begin{array}{c}\Delta V \\
\left(\mathrm{~km} \mathrm{~s}^{-1}\right)\end{array}$ & $\begin{array}{c}V_{\mathrm{p}} \\
\left(\mathrm{km} \mathrm{s}^{-1}\right)\end{array}$ & $\begin{array}{c}S_{\mathrm{p}} \\
(\mathrm{Jy})\end{array}$ & $\begin{array}{c}S_{\mathrm{i}} \\
\left(\mathrm{Jy} \mathrm{km} \mathrm{s}^{-1}\right)\end{array}$ & $\begin{array}{l}\text { Ref. } \\
\text { det. }\end{array}$ & $\begin{array}{c}\Delta \text { pos } \\
(\operatorname{arcsec})\end{array}$ & $\begin{array}{c}\text { IR counterpart } \\
\text { candidate }\end{array}$ \\
\hline $20.08-0.14^{b}$ & 182810.8 & -112844 & & 43.7 & 1.6 & 0.6 & 4 & 8.2 & IRAS $18253-1130$ \\
\hline $20.24+0.07$ & 182745.0 & -111441 & $+60 ;+78$ & 72.0 & 40.6 & 32.8 & 2 & 10.5 & IRAS $18249-1116$ \\
\hline $21.41-0.25$ & 183106.3 & -102137 & $+86 ;+92$ & 89.1 & 5.2 & 5.9 & 8 & & \\
\hline $21.57-0.03$ & 183036.5 & -100644 & $+109 ;+121$ & 117.2 & 8.8 & 13.5 & 8 & 32.7 & IRAS $18278-1009$ \\
\hline $21.87+0.01^{c}$ & 183102.0 & -094903 & $+20 ;+22$ & 20.5 & 2.7 & 1.8 & 2 & 9.6 & IRAS 18282-0951 \\
\hline $22.05+0.22$ & 183035.7 & -093426 & $+44 ;+55$ & 53.8 & 6.1 & 9.1 & 8 & 38.0 & IRAS $18278-0936$ \\
\hline $22.34-0.16$ & 183232.1 & -092910 & $+25 ;+40$ & 35.5 & 28.1 & 22.1 & 1 & 25.2 & IRAS 18297-0931 \\
\hline $22.35+0.06$ & 183145.6 & -092244 & $+79 ;+89$ & 80.1 & 38.7 & 28.1 & 3 & 35.1 & IRAS 18290-0924 \\
\hline $22.45-0.17$ & 183245.8 & -092406 & $+24 ;+40$ & 29.5 & 12.3 & 24.3 & 4 & 37.8 & G022.4595-00.1657 \\
\hline $23.01-0.41$ & 183440.6 & -090028 & $+72 ;+84$ & 74.2 & 188.5 & 431.3 & 4 & 1.8 & $\mathrm{~g} 02301-1^{h}$ \\
\hline $23.19-0.38$ & 183454.5 & -085004 & $+73 ;+83$ & 81.8 & 23.4 & 58.2 & 8 & & \\
\hline $23.26-0.24$ & 183430.8 & -084242 & $+63 ;+66$ & 64.0 & 5.0 & 6.0 & 3 & 52.8 & IRAS 18317-0845 \\
\hline $23.39+0.19$ & 183313.1 & -082356 & $+71 ;+78$ & 74.5 & 19.3 & 35.5 & 8 & 14.7 & IRAS $18305-0826$ \\
\hline $23.44-0.18$ & 183438.8 & -083137 & $+95 ;+113$ & 103.2 & 42.1 & 80.5 & 2 & 11.8 & $\mathrm{~g} 02343-1^{h}$ \\
\hline $23.49+0.08$ & 183347.4 & -082137 & $+81 ;+89$ & 87.1 & 10.7 & 8.2 & 3 & & \\
\hline $23.66-0.13$ & 183451.7 & -081816 & $+75 ;+88$ & 82.3 & 7.8 & 21.6 & 8 & 9.5 & IRAS $18321-0820$ \\
\hline $23.97-0.11$ & 183522.9 & -080111 & $+61 ;+72$ & 71.0 & 13.1 & 10.0 & 8 & 52.3 & G023.9843-00.1117 \\
\hline $24.15-0.01$ & 183520.8 & -074848 & $+16 ;+19$ & 17.6 & 25.8 & 18.4 & 8 & 6.2 & IRAS $18326-0751$ \\
\hline $24.33+0.14$ & 183509.6 & -073453 & $+107 ;+116$ & 110.4 & 7.7 & 9.5 & 2 & 20.4 & IRAS $18324-0737$ \\
\hline $24.51-0.05^{b}$ & 183608.7 & -073055 & $+109 ;+116$ & 114.8 & 14.2 & 14.9 & 5 & 30.0 & IRAS $18334-0733$ \\
\hline $24.53+0.32$ & 183453.0 & -071911 & $+103 ;+111$ & 106.5 & 12.2 & 23.0 & 8 & 46.7 & IRAS $18322-0721$ \\
\hline $24.64-0.33$ & 183725.2 & -073153 & $+35 ;+45$ & 35.4 & 2.3 & 2.9 & 1 & 42.6 & IRAS $18346-0734$ \\
\hline $24.79+0.09^{a}$ & 183611.9 & -071210 & $+106 ;+115$ & 113.6 & 58.5 & 85.9 & 2 & 18.3 & $\mathrm{~g} 02478-1^{h}$ \\
\hline $24.85+0.09^{a}$ & 183617.4 & -070858 & $+109 ;+115$ & 110.0 & 13.8 & 19.4 & 4 & 16.4 & IRAS $18335-0711$ \\
\hline $24.93+0.08^{a}$ & 183629.2 & -070505 & $+46 ;+54$ & 53.4 & 4.2 & 4.4 & 1 & 28.7 & IRAS $18337-0707$ \\
\hline $25.40+0.11$ & 183715.6 & -063905 & $+93 ;+98$ & 94.7 & 3.7 & 5.6 & 5 & 39.8 & IRAS $18345-0641$ \\
\hline $25.71+0.04^{a}$ & 183803.1 & -062432 & $+89 ;+101$ & 95.6 & 473.7 & 414.8 & 6 & 37.5 & $\mathrm{G} 025.7157+00.0487$ \\
\hline $25.83-0.18$ & 183904.7 & -062417 & $+90 ;+100$ & 91.6 & 65.8 & 89.2 & 3 & 44.3 & G025.8197-00.1868 \\
\hline $26.59-0.01^{b}$ & 183952.8 & -053848 & $+22 ;+26$ & 22.9 & 18.4 & 12.1 & 8 & 55.8 & G026.5972-00.0235 \\
\hline $26.60-0.24$ & 184042.1 & -054421 & $+102 ;+116$ & 103.3 & 14.6 & 18.2 & 5 & & \\
\hline $26.65+0.02^{a}$ & 183951.8 & -053452 & & 107.6 & 3.5 & 1.2 & 8 & 22.2 & IRAS 18372-0537 \\
\hline $27.21+0.26$ & 184003.8 & -045809 & $+7 ;+10$ & 9.1 & 2.8 & 3.2 & 1 & 42.0 & $1840000-045806^{f}$ \\
\hline $27.22+0.14$ & 184029.6 & -050109 & $+109 ;+121$ & 118.7 & 9.9 & 20.3 & 1 & 15.3 & $\mathrm{G} 027.2217+00.1362$ \\
\hline $27.28+0.15^{b}$ & 184033.9 & -045718 & $+34 ;+36$ & 34.9 & 23.2 & 13.6 & 7 & 21.4 & IRAS $18379-0500$ \\
\hline $27.36-0.16^{b}$ & 184150.7 & -050145 & $+97 ;+102$ & 100.3 & 18.3 & 20.6 & 4 & 27.7 & IRAS 18391-0504 \\
\hline $27.78+0.07$ & 184147.5 & -043311 & $+112 ;+114$ & 112.3 & 2.4 & 1.8 & 8 & 49.1 & G027.7840+00.0570 \\
\hline $28.02-0.44$ & 184402.1 & -043414 & $+15 ;+28$ & 16.0 & 3.7 & 5.1 & 1 & 33.0 & G028.0245-00.4478 \\
\hline $28.15+0.00$ & 184241.0 & -041521 & $+98 ;+105$ & 101.4 & 58.1 & 27.1 & 4 & 12.8 & G028.1464-00.0008 \\
\hline $28.19-0.05^{b}$ & 184257.8 & -041421 & $+94 ;+102$ & 97.2 & 2.5 & 2.7 & 4 & 35.6 & IRAS $18403-0417$ \\
\hline $28.31-0.40^{a}$ & 184424.9 & -041756 & $+79 ;+94$ & 81.1 & 63.6 & 61.7 & 3 & & \\
\hline $28.40+0.07^{a}$ & 184254.5 & -040004 & $+68 ;+73$ & 68.9 & 4.2 & 5.1 & 8 & 12.8 & IRAS $18402-0403$ \\
\hline $28.53+0.12$ & 184257.7 & -035159 & $+24 ;+28$ & 27.1 & 1.3 & 1.2 & 1 & 25.7 & IRAS $18403-0354$ \\
\hline $28.69+0.41$ & 184213.6 & -033507 & & 94.2 & 4.2 & 0.6 & 1 & 19.2 & IRAS $18396-0337$ \\
\hline $28.82+0.37$ & 184235.2 & -032926 & $+86 ;+93$ & 90.7 & 7.2 & 12.9 & 1 & 18.8 & G028.8174+00.3655 \\
\hline $28.84-0.25$ & 184450.0 & -034523 & $+80 ;+93$ & 83.5 & 72.6 & 122.5 & 2 & 30.1 & IRAS $18421-0348$ \\
\hline $28.84-0.23$ & 184447.1 & -034439 & $+99 ;+104$ & 100.3 & 1.9 & 1.2 & 4 & 54.5 & IRAS $18421-0348$ \\
\hline $28.85+0.50$ & 184212.8 & -032426 & $+80 ;+90$ & 83.5 & 2.7 & 2.1 & 1 & & \\
\hline $29.31-0.15$ & 184523.1 & -031723 & $+43 ;+50$ & 48.7 & 2.8 & 3.1 & 1 & 44.2 & IRAS $18427-0320$ \\
\hline $29.86-0.05$ & 184601.6 & -024526 & $+100 ;+105$ & 101.4 & 52.2 & 71.7 & 4 & 39.5 & G029.8591-00.0608 \\
\hline $29.95-0.02^{b}$ & 184603.8 & -023925 & $+95 ;+102$ & 95.9 & 132.0 & 200.5 & 2 & 25.4 & IRAS $18434-0242$ \\
\hline
\end{tabular}


Table 1. continued.

\begin{tabular}{|c|c|c|c|c|c|c|c|c|c|}
\hline Source $(l, b)$ & $\alpha(2000)$ & $\delta(2000)$ & $\begin{array}{c}\Delta V \\
\left(\mathrm{~km} \mathrm{~s}^{-1}\right)\end{array}$ & $\begin{array}{c}V_{\mathrm{p}} \\
\left(\mathrm{km} \mathrm{s}^{-1}\right)\end{array}$ & $\begin{array}{c}S_{\mathrm{p}} \\
(\mathrm{Jy})\end{array}$ & $\begin{array}{c}S_{\mathrm{i}} \\
\left(\mathrm{Jy} \mathrm{km} \mathrm{s}^{-1}\right)\end{array}$ & $\begin{array}{l}\text { Ref. } \\
\text { det. }\end{array}$ & $\begin{array}{c}\Delta \mathrm{pos} \\
(\operatorname{arcsec})\end{array}$ & $\begin{array}{l}\text { IR counterpart } \\
\text { candidate }\end{array}$ \\
\hline $29.97-0.04$ & 184611.4 & -023914 & $+102 ;+106$ & 103.2 & 23.5 & 30.5 & 4 & & \\
\hline $30.20-0.17$ & 184704.2 & -023036 & $+103 ;+111$ & 108.7 & 22.8 & 30.5 & 2 & 3.9 & G030.1990-00.1693 \\
\hline $30.22-0.19^{a}$ & 184709.8 & -022938 & $+111 ;+115$ & 112.8 & 13.6 & 15.7 & 4 & 39.3 & G030.2212-00.1790 \\
\hline $30.31+0.07$ & 184623.7 & -021756 & $+31 ;+51$ & 36.4 & 8.5 & 17.1 & 8 & & \\
\hline $30.40-0.29$ & 184751.2 & -022315 & $+98 ;+106$ & 98.4 & 5.1 & 7.8 & 1 & & \\
\hline $30.59-0.13$ & 184737.2 & -020846 & & 115.5 & 2.2 & 0.7 & 1 & 19.0 & G030.5945-00.1273 \\
\hline $30.59-0.04^{a}$ & 184718.5 & -020623 & $+40 ;+49$ & 43.1 & 6.2 & 8.2 & 2 & 20.1 & IRAS 18446-0209 \\
\hline $30.70-0.07^{c}$ & 184736.5 & -020111 & $+85 ;+92$ & 88.1 & 94.8 & 60.2 & 4 & 7.1 & FIR 30.70-0.07e \\
\hline $30.76-0.05^{b}$ & 184738.4 & -015705 & $+89 ;+94$ & 91.6 & 71.5 & 72.7 & 4 & 13.1 & IRAS $18450-0200$ \\
\hline $30.78+0.23$ & 184641.2 & -014831 & $+47 ;+50$ & 49.0 & 36.7 & 20.2 & 4 & & \\
\hline $30.79+0.20$ & 184648.0 & -014856 & $+75 ;+90$ & 88.0 & 19.8 & 45.9 & 4 & 18.8 & G030.7951+00.1989 \\
\hline $30.82+0.27$ & 184637.4 & -014514 & $+100 ;+111$ & 110.4 & 3.9 & 5.3 & 2 & 15.8 & IRAS $18440-0148$ \\
\hline $30.82-0.05$ & 184746.2 & -015414 & $+92 ;+109$ & 101.5 & 12.9 & 24.5 & 2 & 22.3 & FIR 30.81-0.05 \\
\hline $30.89+0.17$ & 184707.3 & -014415 & $+98 ;+111$ & 102.0 & 45.1 & 86.7 & 3 & & \\
\hline $30.96-0.14$ & 184819.7 & -014859 & $+74 ;+80$ & 74.8 & 11.3 & 17.3 & 1 & & \\
\hline $31.05+0.35^{a}$ & 184646.2 & -013106 & $+78 ;+82$ & 80.7 & 3.7 & 6.6 & 8 & 17.2 & IRAS 18441-0134 \\
\hline $31.06+0.09^{a}$ & 184742.1 & -013733 & $+15 ;+21$ & 16.3 & 22.0 & 14.5 & 3 & & \\
\hline $31.15+0.05^{a}$ & 184800.3 & -013401 & & 41.2 & 1.9 & 1.2 & 8 & 53.1 & IRAS 18454-0136 \\
\hline $31.27+0.06^{c}$ & 184810.9 & -012644 & $+110 ;+113$ & 110.2 & 69.2 & 148.8 & 2 & 39.7 & $\mathrm{G} 031.2808+00.0618$ \\
\hline $31.41+0.31^{c}$ & 184732.2 & -011249 & $+92 ;+106$ & 96.0 & 11.1 & 36.8 & 3 & 22.0 & IRAS 18449-0115 \\
\hline $31.57+0.06$ & 184844.3 & -011118 & $+95 ;+100$ & 98.8 & 3.1 & 4.6 & 8 & & \\
\hline $32.05+0.06$ & 184937.3 & -004547 & $+92 ;+103$ & 93.2 & 141.2 & 195.7 & 5 & 24.1 & IRAS 18470-0049 \\
\hline $32.74-0.08^{a}$ & 185122.8 & -001215 & $+29 ;+40$ & 38.7 & 58.4 & 85.1 & 2 & 47.9 & IRAS $18487-0015$ \\
\hline $32.98+0.04^{b}$ & 185123.0 & +000346 & $+89 ;+93$ & 92.0 & 30.7 & 23.3 & 5 & 47.7 & IRAS $18488+0000$ \\
\hline $33.10-0.06$ & 185158.9 & +000727 & $+94 ;+105$ & 104.2 & 24.4 & 35.4 & 4 & & \\
\hline $33.13-0.09^{d}$ & 185207.1 & +000756 & $+71 ;+81$ & 73.1 & 16.3 & 19.6 & 2 & 51.9 & IRAS 18496+0004 \\
\hline $33.39+0.03$ & 185210.6 & +002509 & $+97 ;+108$ & 105.0 & 31.0 & 61.3 & 7 & & \\
\hline $33.64-0.21$ & 185328.7 & +003158 & $+58 ;+64$ & 60.3 & 108.7 & 154.6 & 8 & 20.1 & G033.6455-00.2105 \\
\hline $33.74-0.15$ & 185326.9 & +003901 & $+53 ;+55$ & 53.6 & 1.4 & 1.1 & 1 & & \\
\hline $33.86+0.01$ & 185305.2 & +004936 & $+60 ;+65$ & 64.0 & 3.0 & 3.6 & 1 & 40.3 & G033.8707+00.0130 \\
\hline $33.97-0.02$ & 185324.1 & +005513 & $+58 ;+66$ & 59.2 & 3.8 & 7.4 & 8 & & \\
\hline $34.10+0.01$ & 185331.9 & +010226 & $+54 ;+62$ & 55.9 & 4.8 & 5.5 & 1 & 31.1 & G034.0976+00.0183 \\
\hline $34.24+0.13$ & 185321.5 & +011343 & $+54 ;+62$ & 55.4 & 20.8 & 15.1 & 2 & 45.5 & $\mathrm{G} 34.3+0.2 \mathrm{~S}^{g}$ \\
\hline $34.25+0.16^{b}$ & 185316.7 & +011449 & $+57 ;+62$ & 57.8 & 32.4 & 25.8 & 2 & 47.1 & IRAS $18507+0110$ \\
\hline $34.40+0.21$ & 185321.5 & +012409 & $+55 ;+64$ & 55.7 & 19.5 & 18.9 & 3 & & \\
\hline $34.74-0.09$ & 185503.4 & +013417 & $+50 ;+54$ & 52.9 & 9.7 & 9.0 & 1 & & \\
\hline $35.02+0.35^{a}$ & 185400.6 & +020050 & $+40 ;+47$ & 44.2 & 38.9 & 33.8 & 2 & 18.9 & G035.0252+00.3500 \\
\hline $35.79-0.17$ & 185716.1 & +022744 & $+58 ;+65$ & 60.8 & 24.5 & 38.1 & 1 & 26.1 & IRAS $18547+0223$ \\
\hline $36.11+0.55$ & 185515.6 & +030442 & $+69 ;+85$ & 73.0 & 43.3 & 58.4 & 8 & 22.4 & IRAS $18527+0301$ \\
\hline $36.70+0.09$ & 185800.9 & +032330 & $+52 ;+63$ & 53.3 & 8.6 & 9.7 & 1 & 20.5 & IRAS 18554+0319 \\
\hline $37.02-0.03$ & 185859.9 & +033740 & $+77 ;+80$ & 78.8 & 7.3 & 7.1 & 1 & & \\
\hline $37.47-0.11^{a}$ & 190006.7 & +035927 & $+54 ;+64$ & 58.4 & 12.5 & 30.8 & 8 & 23.7 & G037.4688-00.1034 \\
\hline $37.53-0.11^{b}$ & 190014.4 & +040235 & $+48 ;+56$ & 50.0 & 5.8 & 8.8 & 8 & 48.6 & IRAS $18577+0358$ \\
\hline $37.55+0.19$ & 185911.6 & +041208 & $+79 ;+87$ & 83.6 & 8.1 & 12.0 & 7 & 41.0 & IRAS $18566+0408$ \\
\hline $37.60+0.42$ & 185828.5 & +042034 & $+84 ;+93$ & 85.8 & 24.3 & 28.3 & 1 & 15.3 & IRAS $18559+0416$ \\
\hline $38.03-0.30$ & 190150.0 & +042354 & $+55 ;+64$ & 55.7 & 18.6 & 21.6 & 1 & 36.7 & IRAS $18593+0419$ \\
\hline $38.12-0.24^{a}$ & 190147.6 & +043032 & $+68 ;+78$ & 70.0 & 4.2 & 7.3 & 8 & 58.7 & IRAS $18592+0426$ \\
\hline $38.20-0.08$ & 190122.8 & +043910 & $+79 ;+85$ & 79.8 & 8.4 & 12.0 & 1 & 12.8 & G038.1983-00.0830 \\
\hline $38.26-0.08$ & 190128.7 & +044202 & $+6 ;+16$ & 15.4 & 7.9 & 7.6 & 1 & 24.0 & IRAS $18589+0437$ \\
\hline $39.10+0.48$ & 190059.5 & +054228 & $+12+28$ & 15.3 & 15.3 & 23.3 & 1 & 44.6 & IRAS $18585+0538$ \\
\hline
\end{tabular}

Masers associated with UCHII regions (Becker et al. 1994; Walsh et al. 1998; Lockman 1989): ${ }^{a}$ weak source at 5 GHz, ${ }^{b}$ bright source at 1.4 and $5 \mathrm{GHz},{ }^{c}$ continuum source with $S_{1.4 \mathrm{GHz}}>S_{5 \mathrm{GHz}},{ }^{d}$ source of recombination lines.

Reference of the first detection: 1) this paper, 2) Menten (1991), 3) Schutte et al. (1993), 4) Caswell et al. (1995), 5) van der Walt et al. (1995), 6) Walsh et al. (1997), 7) Slysh et al. (1999), 8) Szymczak et al. (2000)

Infrared identifications; ${ }^{e}$ Lester et al. (1985), ${ }^{f}$ Skrutskie et al. (1997), ${ }^{g}$ ISO (2001), ${ }^{h}$ Testi et al. (1998). 

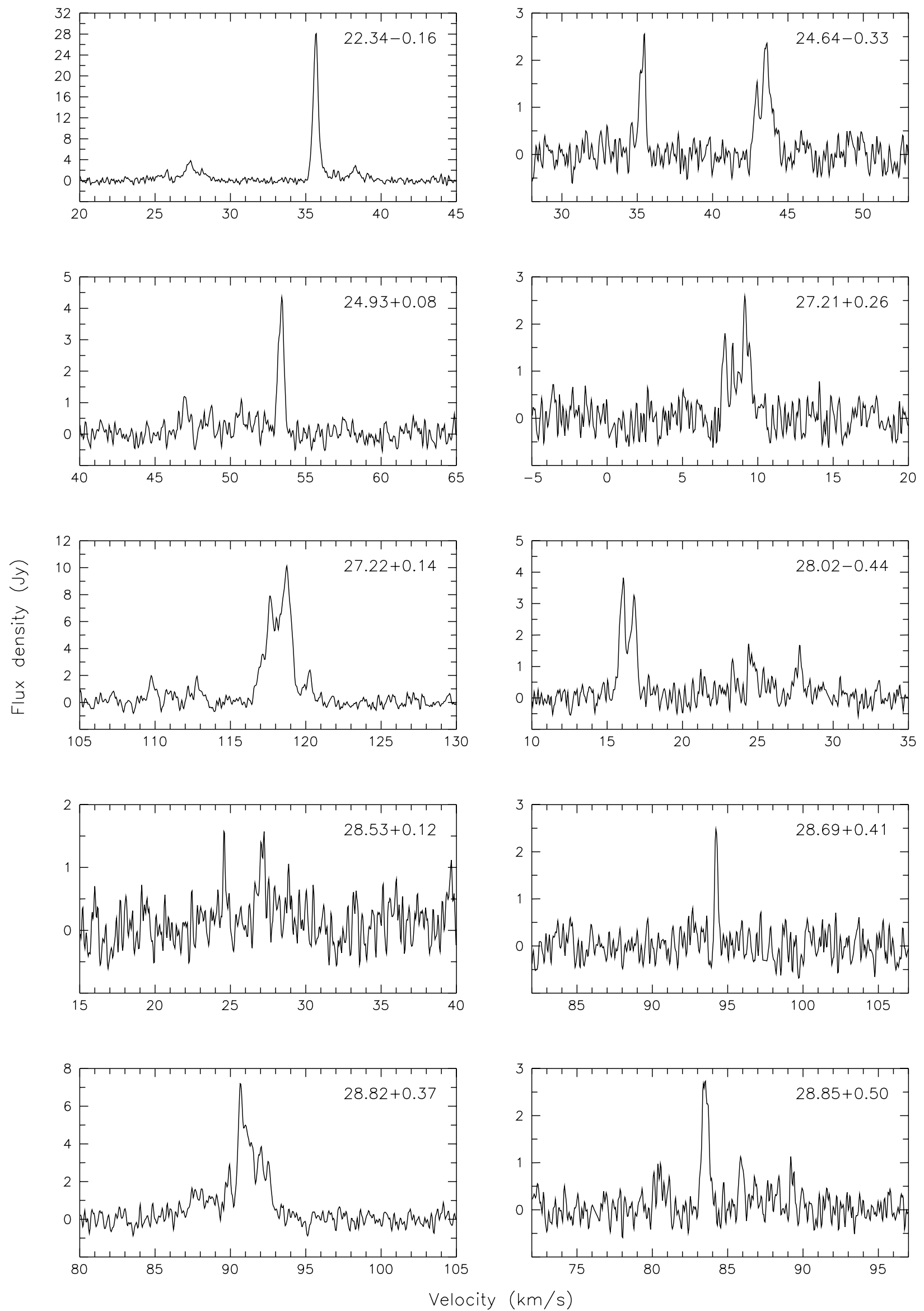

Fig. 1. The 6.7 GHz spectra of the newly detected methanol sources. 

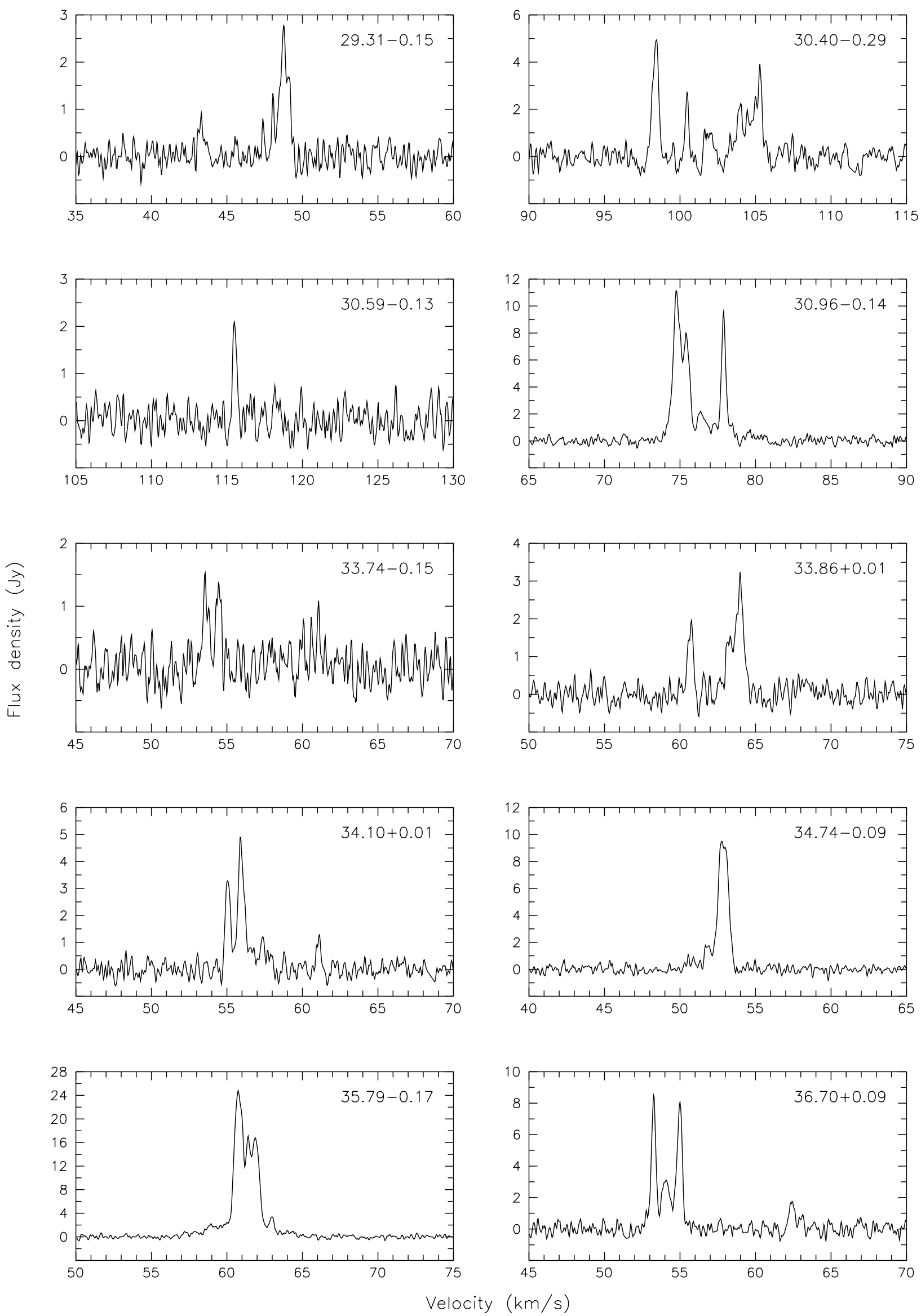

Fig. 1. continued. 

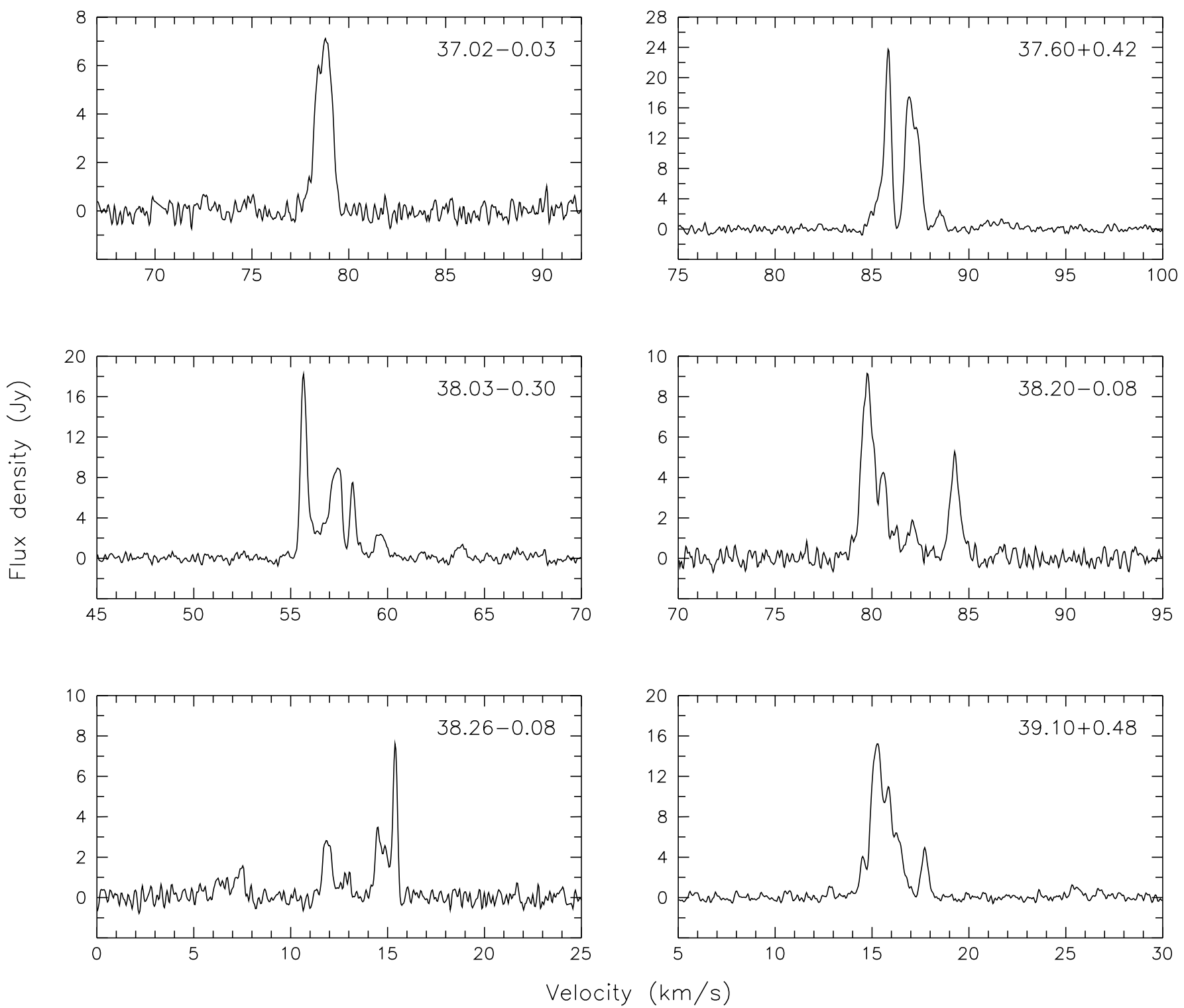

Fig. 1. continued.

one source IRAS $18305-0826$ that has colours slightly outside the HM89 limits. We conclude that in the surveyed strip of the Galactic plane not all the IRAS sources associated with the $6.7 \mathrm{GHz}$ masers have colours typical for UCHII regions.

In the surveyed area there are 209 IRAS sources with a flux density at $60 \mu \mathrm{m}$ greater than $20 \mathrm{Jy}$ and colours satisfying WC criteria. This implies $\sim 13 \%$ (27/209) probability of the detection of $6.7 \mathrm{GHz}$ maser emission. The probability of $\mathrm{CH}_{3} \mathrm{OH}$ maser association slightly increases to $16 \%$ (17/106) for objects of high quality IRAS measurements at all 12, 25 and $60 \mu \mathrm{m}$ bands.

Within the surveyed area 21 masers do not have IR counterparts within $1^{\prime}$ from their positions given in Table 1. Figure 3 shows a histogram of the galactic longitude distribution of these sources. The number of methanol sources reaches a maximum near the galactic longitude of about $30^{\circ}$. This enhancement appears within $\sim 4^{\circ}$ line of sight that is tangential to the
Scutum spiral arm as determined by Georgelin \& Georgelin (1976). Because of uncertain kinematic distances it does not implicate unambiguously the $6.7 \mathrm{GHz}$ methanol emission as a tracer of spiral arms. For $30^{\circ}<l<35^{\circ}$ the relative number of methanol sources without IR associations significantly increases. A plausible cause is that many sources were not included in the IRAS catalogue as their flux density measurements and positions were severely confused due to a large number of sources and/or diffuse emission.

\subsection{Radio continuum sources}

There are 32 methanol sources, with names marked in Table 1, which lie within $1^{\prime}$ of the UCHII region recognized by radio continuum emission (Becker et al. 1994; Walsh et al. 1998) or radio recombination lines (Lockman 1989). Object 33.13-0.09 has been found as an emitter of recombination lines 


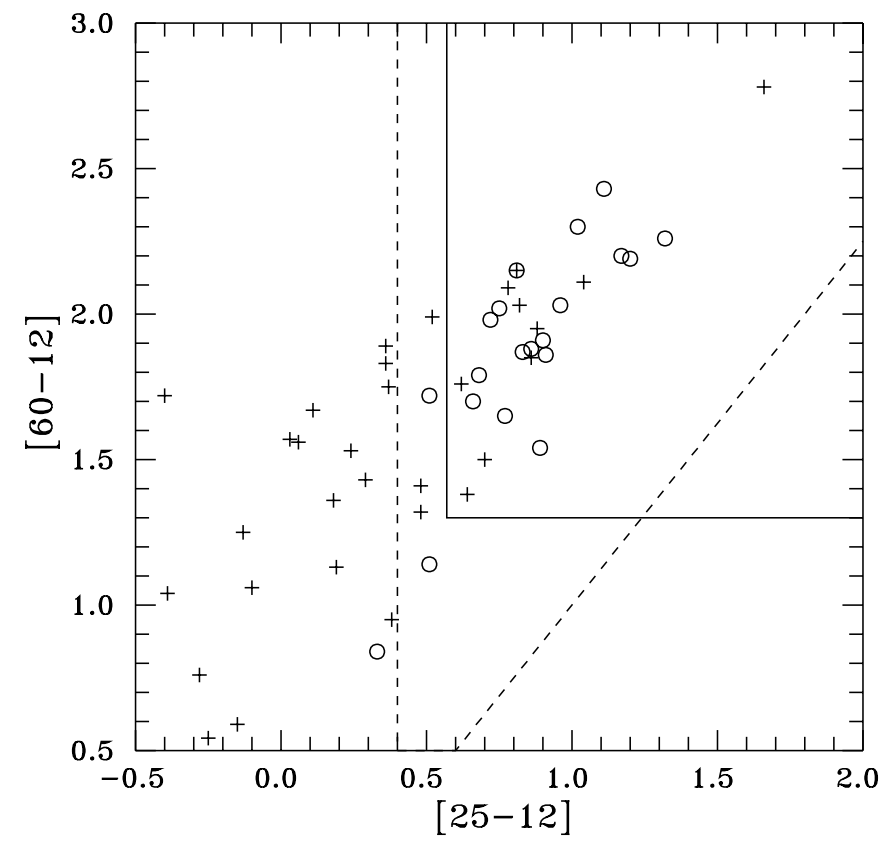

Fig. 2. Colour-colour diagram for all IRAS sources associated with the $6.7 \mathrm{GHz}$ methanol maser emission detected in the survey. The circles and crosses indicate the sources with well determined colours and poorly determined or undetermined colours, respectively. Solid and dashed lines delineate the WC89 and HM89 limits for UCHII regions, respectively.

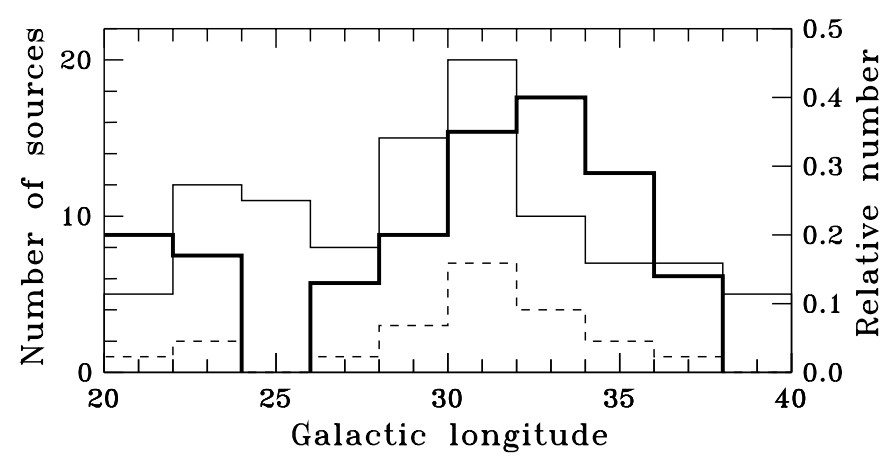

Fig. 3. Distribution in galactic longitude of the all detected methanol maser sources (solid line) and the sources without IR counterparts (dashed line). Relative number of the sources that have not IR counterparts is shown by thick solid line.

(Lockman 1989) but no radio continuum was detected by Becker et al. (1994). 16 out of 31 objects are weak continuum sources with a mean flux density of $12 \mathrm{mJy}$ at $5 \mathrm{GHz}$ and were not detected at $1.4 \mathrm{GHz}$ (Becker et al. 1994). These are very likely to be primary UCHII regions. A further 11 out of 31 objects are bright sources $(\geq 50 \mathrm{mJy}$ at $5 \mathrm{GHz}$ ) with optically thick thermal emission. The other 4 objects having continuum flux at $1.4 \mathrm{GHz}$ higher than that at $5 \mathrm{GHz}$ are likely to be optically thin. The statistics of the occurrence of $6.7 \mathrm{GHz}$ methanol masers associated with UCHII regions is meaningful as our blind survey results are compared with the VLA complete survey at $5 \mathrm{GHz}$ of angular resolution of about $5^{\prime \prime}$ and a sensitivity of 2.5-10 mJy (Becker et al. 1994). We can conclude that about one third of our methanol sources are associated with UCHII regions of $5 \mathrm{GHz}$ flux density ranging from 3.4 to $1127 \mathrm{mJy}$.

\subsection{CO emission}

Figure 4 is the galactic longitude - radial velocity diagram for the $6.7 \mathrm{GHz}$ methanol sources superimposed on the CO emission map (Dame et al. 2001). With the exception of a few sources of a single emission feature the velocity plotted corresponds to the central velocity of the maser emission range rather than the velocity of the peak emission. The plot shows that only 13 sources have radial velocities lower than $40 \mathrm{~km} \mathrm{~s}^{-1}$ while sources with radial velocities higher than $70 \mathrm{~km} \mathrm{~s}^{-1}$ dominate. This implies that the majority of the methanol sources have the velocities in the range typical of the molecular ring (Cohen et al. 1986).

The distribution of masers in the $l-V$ diagram is not uniform; for large areas covered by $\mathrm{CO}$ emission there are no methanol sources at all, while for others we observe clusters of various sizes. About $35 \%$ of sources are in clusters of up to 3-6 objects characterized by narrow ranges of galactic coordinates $\left(<0.4^{\circ}\right)$ and velocity $\left(<10 \mathrm{~km} \mathrm{~s}^{-1}\right)$. A prominent cluster of 6 masers in the vicinity of the bright UCHII region G34.25+0.14 is located in a giant molecular cloud (Scoville et al. 1987) and has a linear extent of about 40 pc. We suggest that the $l-V$ diagram of methanol sources reflects a clustered mode of massive star formation (Stahler et al. 2000).

\section{Discussion}

This blind survey yielded a number of detections per area bin very similar to that reported by Ellingsen et al. (1996b) for region $325^{\circ}<l<335^{\circ}$ and $|b| \leq 0.53$. In the longitude range of $25^{\circ}<l<35^{\circ}$ we detected 61 sources while in the area in the fourth quadrant Ellingsen et al. found 50 sources. As the sensitivity limits of both surveys were similar we conclude that the probability of detecting methanol emission in the two galactic regions is comparable.

In the surveyed area, 5 known methanol sources were not detected. The sources IRAS 18305-0758, IRAS 18321-0843, IRAS 18341-0727 and IRAS 18403-0445 were detected by Szymczak et al. (2000) and 28.86+0.07 was detected by Caswell et al. (1995). All but one (IRAS 18305-0758) are weak masers with $S_{\mathrm{p}}<3.5 \mathrm{Jy}$. Apparently, their emission diminished below our sensitivity limit after about one year.

In the surveyed area about half of the masers do not have IRAS counterpart candidates within $1^{\prime}$. Thus, the observations of IRAS selected samples underestimate the maser number by a factor of about two. This supports the results obtained by Ellingsen et al. (1996b). We have demonstrated convincingly that the number of unassociated masers significantly increases in the region where IRAS measurements are expected to be seriously affected by confusion. Searching for IR counterparts of methanol sources in various catalogues has further decreased the number of unassociated masers. We have noticed that only 6 sources do not have IR counterparts within 1.5. They are usually weak methanol masers whose positions have 


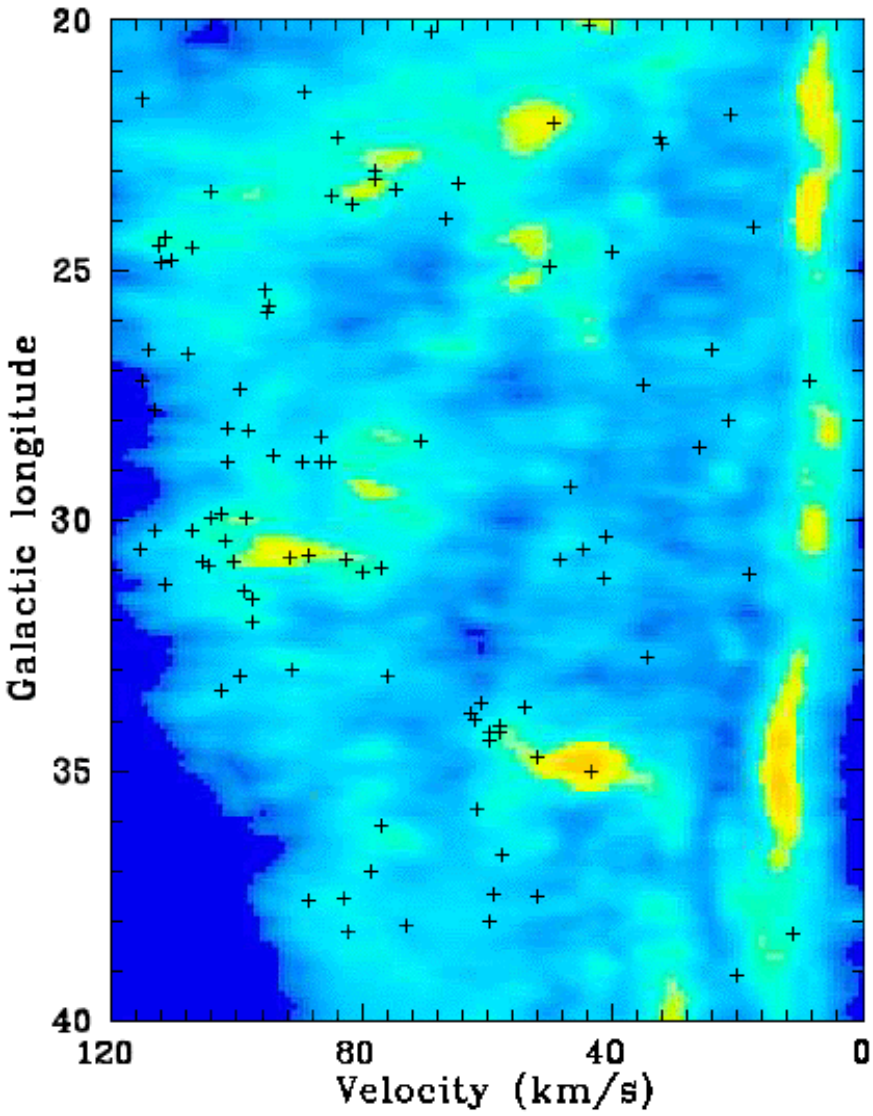

Fig. 4. Distribution of the radial velocity of methanol sources denoted by crosses as a function of galactic longitude, superimposed on the velocity distribution of the $\mathrm{CO}(J=1-0)$ emission taken from Dame et al. (2001).

been poorly determined. Astrometric observations are highly desirable to verify whether these sources are really isolated.

Among the IRAS sources of well determined flux densities and associated with the methanol emission almost all have colours typical of UCHII regions as established by WC and MH criteria. For the IRAS sources with poorly determined colours we cannot assume, however, that all of these are related to the UCHII regions. A significant deficit of $\mathrm{CH}_{3} \mathrm{OH}$ sources associated with the observed radio continuum emission was demonstrated when we searched for $5 \mathrm{GHz}$ radio counterparts in Becker et al.'s (1994) catalogue. About two thirds of the methanol masers are not associated with radio continuum emission within 1'. Because our sample is flux density limited, we suggest that some sources are distant objects and their continuum emission is too weak to be detected in the VLA survey of 2.5-10 mJy sensitivity. However, for several sources we must assume the near kinematic distances because the far ones imply unrealistically high maser luminosity. In those cases the lack of $5 \mathrm{GHz}$ emission is not a sensitivity effect. The present estimate of the probability that methanol sources are associated with detectable HII regions is consistent with the finding by Walsh et al. (1998) who detected, with a sensitivity of about $1 \mathrm{mJy}$, radio continuum emission only in about $20 \%$ of the sample of 233 methanol sources. Phillips et al. (1998) noted a significantly higher success rate of $56 \%$ while observing the $8.6 \mathrm{GHz}$ emission with a detection limit of about $0.5 \mathrm{mJy}$ towards 45 methanol sources.

\section{Conclusions}

The blind survey for $6.7 \mathrm{GHz}$ methanol emission has yielded 100 sources, of which 26 objects have not been catalogued before.

$21 \%$ of methanol masers do not have infrared counterpart candidates within a $1^{\prime}$ radius. The number of unassociated masers increases along the direction nearly tangent to the Scutum spiral arm $\left(30^{\circ}<l<35^{\circ}\right)$ very likely due to the exclusion of some sources from the IRAS catalogue caused by the large number of objects and the confusion of flux density measurements. Almost all IRAS objects with flux density measurements of good quality, unambiguously associated with methanol masers have colours typical of UCHII regions.

We have confirmed that the probability of $6.7 \mathrm{GHz}$ emission detection in the IRAS selected samples is $13-16 \%$. Furthermore, the IRAS-based searches underestimate the number of methanol sources by a factor of two.

The comparison of our data with the VLA complete survey maps at $5 \mathrm{GHz}$ has revealed that about one third of methanol masers are associated with UCHII regions. The accurate positions of methanol sources could be used as a final check of this result.

Acknowledgements. We thank T. Dame for providing us with the maps of the CO survey. This research has made use of the SIMBAD database. We are grateful for $\mathrm{KBN}$ support through grants 2P03D01415 and 2P03D01122.

\section{References}

Becker, R. H., White, R. L., Helfand, D. J., \& Zoonematkermani, S. 1994, ApJS, 91, 347

Caswell, J. L. 1996, MNRAS, 279, 79

Caswell, J. L., Vaile, R. A., Ellingsen, S. P., Whiteoak, J. B., \& Norris, R. P. 1995, MNRAS, 272, 96

Cohen, R. S., Dame, T. M., \& Thaddeus, P. 1986, ApJS, 60, 695

Dame, T. M., Hartmann, D., \& Thaddeus, P. 2001, ApJ, 547, 792

Egan, M. P., Price, S. D., Moshir, M. M., et al. 1999, The Midcourse Space Experiment Point Source Catalog, http://vizier.u-strasbg.fr/viz-bin/Cat?V/107

Ellingsen, S. P., Norris, R. P., \& McCulloch, P. M. 1996a, MNRAS, 279, 101

Ellingsen, S. P., von Bibra, M. L., McCulloch, P. M., et al. 1996b, MNRAS, 280, 378

Georgelin, Y. M., \& Georgelin, Y. P. 1976, A\&A, 49, 57

Hughes, V. A., \& MacLeod, G. C. 1989, AJ, 97, 786

IRAS catalogue of Point Sources, 1986, Joint IRAS Science W.G., http://vizier.u-strasbg.fr/viz-bin/VizieR?source $=\mathrm{II} / 125$

ISO Observation Log of validated data, 2001, ISO Data Centre, http://vizier.u-strasbg.fr/viz-bin/VizieR?source $=\mathrm{B} /$ iso

Johansson, L. E. B., Andersson, C., Goss, W. M., \& Winberg, A. 1977, A\&A, 54, 323

Lester, D. F., Dinerstein, H. L., Werner, M. W., et al. 1985, ApJ, 296, 565 
Lockman, F. J. 1989, ApJS, 71, 469

MacLeod, G. C., van der Walt, D. J., North, A., et al. 1998, AJ, 116, 2936

Menten, K. M. 1991, ApJ, 380, L75

Ott, M., Witzel, A., Quirrenbach, A., et al. 1994, A\&A, 284, 331

Phillips, C. J., Norris, R. P., Ellingsen, S. P., \& McCulloch, P. M. 1998, MNRAS, 300, 1131

Schutte, A. J., van der Walt, D. J., Gaylard, M. J., \& MacLeod, G. C. 1993, MNRAS, 261, 783

Scoville, N. Z., Yun, M. S., Clemens, D. P., Sanders, D. B., \& Waller, W. H. 1987, ApJS, 63, 821

Skrutskie, M. F., Schneider, S. E., Stiening, R., et al. 1997, The Two Micron All Sky Survey, http://vizier.u-strasbg.fr/ cgi-bin/VizieR?-source=B/2mass

Slysh, V. I., Val'tts, I. E., Kalenskii, S. V., et al. 1999, A\&AS, 134 115
Stahler, S. W., Palla, F., \& Ho, P. T. P. 2000, in Protostars and Planets IV, ed. V. Mannings, A. P. Boss, \& S. S. Russell (Univ. of Arizona Press), 327

Szymczak, M., Hrynek, G., \& Kus, A. J. 2000, A\&AS, 143, 269

Szymczak, M., \& Kus, A. J. 2000, A\&A, 360, 311

Testi, L., Felli, M., Persi, P., \& Roth, M. 1998, A\&AS, 129, 495

van der Walt, D. J., Gaylard, M. J., \& MacLeod, G. C. 1995, A\&AS, 110,81

van der Walt, D. J., Retief, S. J. P., Gaylard, M. J., \& MacLeod, G. C. 1996, MNRAS, 282, 1085

Walsh, A. J., Burton, M. G., Hyland, A. R., \& Robinson, G. 1998, MNRAS, 301, 640

Walsh, A. J., Hyland, A. R., Robinson, G., \& Burton, M. G. 1997, MNRAS, 291, 261

Wood, D. O. S., \& Churchwell, E. 1989, ApJ, 340, 265 